\title{
CYCLOTOMIC UNITS AND GREENBERG'S CONJECTURE FOR REAL QUADRATIC FIELDS
}

\author{
TAKASHI FUKUDA
}

Dedicated to Professor Hisashi Ogawa on his 70th birthday

\begin{abstract}
We give new examples of real quadratic fields $k$ for which the Iwasawa invariant $\lambda_{3}(k)$ and $\mu_{3}(k)$ are both zero by calculating cyclotomic units of real cyclic number fields of degree 18 .
\end{abstract}

\section{INTRODUCTION}

Let $k$ be a real quadratic field and $p$ an odd prime number which splits in $k$. Two integers $n_{0}^{(r)}$ and $n_{2}^{(r)}$, which are invariants of $k$, were defined in [6], and numerical results of $n_{0}^{(1)}$ and $n_{2}^{(1)}$ for $p=3$ were given in [2]. Using these data, we verified in [2] Greenberg's conjecture of the case $p=3$ for $2227 k$ 's, where $k=\mathbb{Q}(\sqrt{m})$ and $m$ is a positive square-free integer less than 10000. In this paper, we verify the conjecture for 34 of the remaining 52 fields $k$ in the above range, using $n_{0}^{(2)}$ and $n_{2}^{(2)}$.

We start with the definitions of $n_{0}^{(r)}$ and $n_{2}^{(r)}$. Throughout this paper, $\mu$ denotes the fundamental unit of a real quadratic field $k$. Let $(p)=\mathfrak{p p}^{\prime}$ be the prime decomposition of $p$ in $k$. Let $k_{r}$ be the $r$ th layer of the cyclotomic $\mathbb{Z}_{p}$-extension of $k$, and $\mathfrak{p}_{r}$ the unique prime ideal of $k_{r}$ lying over $\mathfrak{p}$. Let $d_{r}$ be the order of $\operatorname{cl}\left(\mathfrak{p}_{r}\right)$ in the ideal class group of $k_{r}$, and take a generator $\alpha_{r} \in k_{r}$ of $\mathfrak{p}_{r}^{d_{r}}$. First we define $n_{2}$ by

$$
\mathfrak{p}^{\prime n_{2}} \|\left(\mu^{p-1}-1\right)
$$

and next define $n_{0}^{(r)}$ and $n_{2}^{(r)}$ by

$$
\mathfrak{p}^{\prime n_{0}^{(r)}} \|\left(N_{k_{r} / k}\left(\alpha_{r}\right)^{p-1}-1\right), \quad p^{n_{2}^{(r)}}=p^{n_{2}}\left(E(k): N_{k_{r} / k}\left(E\left(k_{r}\right)\right)\right) .
$$

Here, $E(K)$ denotes the unit group of an algebraic number field $K$. We need the inequality $n_{0}^{(r)} \leq n_{2}^{(r)}$ for the uniqueness of $n_{0}^{(r)}$. Note that $n_{2}=n_{2}^{(0)}$. We put $n_{0}=n_{0}^{(0)}$. Moreover, we denote by $A_{r}$ the $p$-Sylow subgroup of the ideal class group of $k_{r}$ and put $D_{r}=\left\langle\operatorname{cl}\left(\mathfrak{p}_{r}\right)\right\rangle \cap A_{r}$.

From now on, we let $p=3$. In order to calculate $n_{0}^{(2)}$ and $n_{2}^{(2)}$, we have to obtain a generator $\alpha_{2}$ of $\mathfrak{p}_{2}^{d_{2}}$ and the group index $\left(E(k): N_{k_{2} / k}\left(E\left(k_{2}\right)\right)\right)$. Since $k_{2}$ is a

Received by the editor January 10, 1995.

1991 Mathematics Subject Classification. Primary 11R23, 11R11, 11R27, 11Y40.

Key words and phrases. Iwasawa invariants, real quadratic fields, unit groups, computation.

(C)1996 American Mathematical Society 
field of degree 18 , we need study the structure of $E\left(k_{2}\right)$ to get them in a reasonable amount of computer time.

\section{Relative units of $k_{2}$}

It is difficult to get a system of fundamental units of $k_{2}$. So we consider the subgroup $E_{R}=\left\{\varepsilon \in E\left(k_{2}\right) \mid N_{k_{2} / \mathbb{Q}_{2}}(\varepsilon)= \pm 1, N_{k_{2} / k}(\varepsilon)= \pm 1\right\}$ of $E\left(k_{2}\right)$, which we call the relative unit group of $k_{2}$. Here, $\mathbb{Q}_{2}=\mathbb{Q}(\cos (2 \pi / 27))$ is the second layer of the $\mathbb{Z}_{3}$-extension of $\mathbb{Q}$.

Lemma 2.1. The free rank of $E_{R}$ is 8 .

Proof. Let $\varepsilon$ be any element of $E\left(k_{2}\right)$. Then

$$
\varepsilon^{18} N_{k_{2} / \mathbb{Q}_{2}}(\varepsilon)^{-9} N_{k_{2} / k}(\varepsilon)^{-2} \in E_{R}
$$

Hence, $E\left(k_{2}\right)^{18} \subset E_{R} E\left(\mathbb{Q}_{2}\right) E\left(k_{2}\right) \subset E\left(k_{2}\right)$. Since $E_{R} \cap E\left(\mathbb{Q}_{2}\right) E(k)=E(\mathbb{Q})$, we see that $\operatorname{rank}\left(E_{R}\right)=\operatorname{rank}\left(E\left(k_{2}\right)\right)-\operatorname{rank}\left(E\left(\mathbb{Q}_{2}\right)\right)-\operatorname{rank}(E(k))=8$.

We fix a generator $\sigma$ of the Galois group $G\left(k_{2} / \mathbb{Q}\right)$ and put $\alpha_{i}=\alpha^{\sigma^{i}}$ for $\alpha \in$ $E\left(k_{2}\right)$.

Lemma 2.2. For $\varepsilon \in E_{R}$, we have $\varepsilon_{8}= \pm\left(\varepsilon_{1} \varepsilon_{3} \varepsilon_{5} \varepsilon_{7}\right)\left(\varepsilon_{0} \varepsilon_{2} \varepsilon_{4} \varepsilon_{6}\right)^{-1}$.

Proof. Since $N_{k_{2} / \mathbb{Q}_{2}}(\varepsilon)=\varepsilon_{0} \varepsilon_{9}= \pm 1$, we have $\varepsilon_{9}= \pm \varepsilon_{0}^{-1}$. Therefore, $N_{k_{2} / k}(\varepsilon)=$ $\varepsilon_{0} \varepsilon_{2} \cdots \varepsilon_{16}= \pm\left(\varepsilon_{0} \varepsilon_{2} \varepsilon_{4} \varepsilon_{6}\right) \varepsilon_{8}\left(\varepsilon_{1} \varepsilon_{3} \varepsilon_{5} \varepsilon_{7}\right)^{-1}= \pm 1$. From this we have the desired relation.

Now, we assume that there exists $\varphi \in E_{R}$ such that $E_{R}=\left\langle-1, \varphi_{0}, \varphi_{1}, \ldots, \varphi_{7}\right\rangle$ and put

$$
\Phi=\varphi_{0} \varphi_{1}^{-2} \varphi_{2}^{3} \varphi_{3}^{-4} \varphi_{4}^{5} \varphi_{5}^{-6} \varphi_{6}^{7} \varphi_{7}^{-8} .
$$

The following property of $\Phi$ is important in our computation.

Lemma 2.3. Let $\varepsilon \in E_{R}$. Then $\varepsilon^{1+\sigma} \in E_{R}^{9}$ if and only if $\varepsilon \equiv \Phi^{i}\left(\bmod E_{R}^{9}\right)$ for some $0 \leq i \leq 8$.

Proof. We can write $\varepsilon= \pm \varphi^{e_{0}} \varphi_{1}^{e_{1}} \cdots \varphi_{7}^{e_{7}}$ with suitable integers $e_{i}$. Then, from Lemma 2.2,

$$
\varepsilon^{1+\sigma}= \pm \varphi_{0}^{e_{0}-e_{7}} \varphi_{1}^{e_{0}+e_{1}+e_{7}} \varphi_{2}^{e_{1}+e_{2}-e_{7}} \cdots \varphi_{6}^{e_{5}+e_{6}-e_{7}} \varphi_{7}^{e_{6}+2 e_{7}} .
$$

It is easily seen that $\left\{\varphi_{0}, \ldots, \varphi_{7}\right\}$ becomes a basis of $E_{R} /\{ \pm 1\}$ if $E_{R}=\left\langle-1, \varphi_{0}\right.$, $\left.\ldots, \varphi_{7}\right\rangle$. Hence, $\varepsilon^{1+\sigma} \in E_{R}^{9}$ if and only if $e_{0}-e_{7} \equiv e_{0}+e_{1}+e_{7} \equiv e_{1}+e_{2}-e_{7} \equiv$ $\cdots \equiv e_{6}+2 e_{7} \equiv 0(\bmod 9)$. This is equivalent to $e_{0} \equiv e_{7}, e_{1} \equiv-2 e_{7}, e_{2} \equiv$ $3 e_{7}, \ldots, e_{6} \equiv 7 e_{7}(\bmod 9)$. Since $e_{7} \equiv-8 e_{7}(\bmod 9)$, we have that $\varepsilon^{1+\sigma} \in E_{R}^{9}$ if and only if $\varepsilon \equiv \Phi^{e^{7}}\left(\bmod E_{R}^{9}\right)$.

\section{Cyclotomic units of $\mathbb{Q}_{2}$}

In this section, we study properties of cyclotomic units of $\mathbb{Q}_{2}$.

First, we treat a more general situation. Let $p$ be an odd prime number and $\theta=\zeta_{p^{n}}+\zeta_{p^{n}}^{-1}$ for a nonnegative integer $n$, where $\zeta_{p^{n}}$ denotes a primitive $p^{n}$ th root of unity. Let $K=\mathbb{Q}(\theta)$ and $r=[K: \mathbb{Q}]$. Then $p$ is fully ramified in $K / \mathbb{Q}$ and $2-\theta$ a generator of the prime ideal of $K$ lying over $p$. Therefore, $(2-\theta)^{r}=p \varepsilon$ for some unit $\varepsilon$ of $K$. We can write $\varepsilon$ explicitly in terms of the conjugates of $\theta$ under a certain condition. 
Lemma 3.1. Assume that 2 is a primitive root modulo $p^{n}$ and let $\sigma$ be the generator of the Galois group $G\left(\mathbb{Q}\left(\zeta_{p^{n}}\right) / \mathbb{Q}\right)$ such that $\zeta_{p^{n}}^{\sigma}=\zeta_{p^{n}}^{2}$. Put $r=p^{n-1}(p-1) / 2$ and $\theta_{i}=\theta^{\sigma^{i}}$. Then

$$
\left(2-\theta_{0}\right)^{r}=p \theta_{0}^{2} \theta_{1}^{4} \cdots \theta_{r-2}^{2(r-1)}
$$

Proof. We put $\zeta=\zeta_{p^{n}}$. Let

$$
f(X)=X^{p^{n-1}(p-1)}+X^{p^{n-1}(p-2)}+\cdots+X^{p^{n-1}}+1=\prod_{1 \leq i \leq 2 r}\left(X-\zeta^{2^{i}}\right)
$$

be the minimal polynomial of $\zeta$ over $\mathbb{Q}$. Since $2^{r} \equiv-1\left(\bmod p^{n}\right)$, we have $\theta_{r}=\theta_{0}$. So we consider the indices $i$ of $\theta_{i}$ modulo $r$. Then

$$
\begin{aligned}
1=f(-1) & =\prod_{1 \leq i \leq 2 r}\left(1+\zeta^{2^{i}}\right) \\
& =\prod_{1 \leq i \leq 2 r} \zeta^{2^{i-1}}\left(\zeta^{2^{i-1}}+\zeta^{-2^{i-1}}\right) \\
& =\left(\prod_{0 \leq i \leq r-1} \theta_{i}\right)^{2}
\end{aligned}
$$

because $\sum_{1 \leq i \leq 2 r} 2^{i-1} \equiv 0\left(\bmod p^{n}\right)$. Therefore, $\theta_{-1}^{2}=\left(\theta_{0} \theta_{1} \cdots \theta_{r-2}\right)^{-2}$. Moreover,

$$
\begin{aligned}
p=f(1) & =\prod_{0 \leq i \leq 2 r-1}\left(1-\zeta^{2^{i}}\right) \\
& =\prod_{0 \leq i \leq r-1}\left(1-\zeta^{2^{i}}\right)\left(1-\zeta^{-2^{i}}\right) \\
& =\prod_{0 \leq i \leq r-1}\left(2-\theta_{i}\right)
\end{aligned}
$$

Now,

$$
\begin{aligned}
2+\theta_{0} & =(1+\zeta)\left(1+\zeta^{-1}\right) \\
& =\zeta^{-2^{r-1}}\left(\zeta^{2^{r-1}}+\zeta^{-2^{r-1}}\right) \zeta^{2^{r-1}}\left(\zeta^{-2^{r-1}}+\zeta^{2^{r-1}}\right) \\
& =\theta_{-1}^{2}
\end{aligned}
$$

Therefore, $2-\theta_{i}=2-\left(\theta_{i-1}^{2}-2\right)=\left(2-\theta_{i-1}\right)\left(2+\theta_{i-1}\right)=\left(2-\theta_{i-1}\right) \theta_{i-2}^{2}$ for all $i$. Hence, we have

$$
\begin{aligned}
2-\theta_{i} & =\left(2-\theta_{i-1}\right) \theta_{i-2}^{2} \\
& =\left(2-\theta_{i-2}\right) \theta_{i-3}^{2} \theta_{i-2}^{2} \\
& =\left(2-\theta_{i-3}\right) \theta_{i-4}^{2} \theta_{i-3}^{2} \theta_{i-2}^{2}
\end{aligned}
$$


for all $i$. Substituting $i=0,1, \ldots, r-1$ in these relations, we have

$$
\begin{aligned}
2-\theta_{0} & =2-\theta_{0}, \\
2-\theta_{1} & =\left(2-\theta_{0}\right) \theta_{-1}^{2}, \\
2-\theta_{2} & =\left(2-\theta_{0}\right) \theta_{-1}^{2} \theta_{0}^{2}, \\
\vdots & \\
2-\theta_{r-1} & =\left(2-\theta_{0}\right) \theta_{-1}^{2} \theta_{0}^{2} \cdots \theta_{r-3}^{2} .
\end{aligned}
$$

Hence, we get

$$
\begin{aligned}
p & =\left(2-\theta_{0}\right)^{r} \theta_{-1}^{2 r-2} \theta_{0}^{2 r-4} \cdots \theta_{r-3}^{2} \\
& =\left(2-\theta_{0}\right)^{r}\left(\theta_{0} \theta_{1} \cdots \theta_{r-2}\right)^{-(2 r-2)} \theta_{0}^{2 r-4} \cdots \theta_{r-3}^{2} \\
& =\left(2-\theta_{0}\right)^{r} \theta_{0}^{-2} \theta_{1}^{-4} \cdots \theta_{r-2}^{-2(r-1)} .
\end{aligned}
$$

We apply Lemma 3.1 to the case $p=3$ and $n=3$. Let $\theta=\zeta_{27}+\zeta_{27}^{-1}$ and put

$$
\Theta=\theta_{0} \theta_{1}^{2} \theta_{2}^{3} \theta_{3}^{4} \theta_{4}^{5} \theta_{5}^{6} \theta_{6}^{7} \theta_{7}^{8} .
$$

Then we have the following corollary.

Corollary 3.2. There holds $3 \Theta^{2} \in \mathbb{Q}_{2}^{9}$.

We need one more property of $\Theta$.

Lemma 3.3. There holds $\Theta^{1-\sigma} \in E\left(\mathbb{Q}_{2}\right)^{9}$.

Proof. As we have seen in the proof of Lemma 3.1, $\theta_{8}^{2}=\left(\theta_{0} \theta_{1} \cdots \theta_{7}\right)^{-2}$. Therefore, $\theta_{8}= \pm\left(\theta_{0} \theta_{1} \cdots \theta_{7}\right)^{-1}$. Hence, $\Theta^{1-\sigma}=\left(\theta_{0} \theta_{1}^{2} \cdots \theta_{7}^{8}\right)\left(\theta_{1} \theta_{2}^{2} \cdots \theta_{8}^{8}\right)^{-1}=$ $\theta_{0} \theta_{1} \cdots \theta_{7} \theta_{8}^{-8}= \pm \theta_{8}^{-9}$.

\section{Computational method For $n_{0}^{(2)}$ And $n_{2}^{(2)}$}

In this section, we explain how to determine $n_{0}^{(2)}$ and $n_{2}^{(2)}$ under the condition $A_{0}=D_{0}$. We can determine $n_{2}^{(2)}$ from (1) if we know the group index $(E(k)$ : $\left.N_{k_{2} / k}\left(E\left(k_{2}\right)\right)\right)$. On the other hand, we see that

$$
\left|D_{r}\right|=\left|A_{0}\right| \frac{p^{r}}{\left(E(k): N_{k_{r} / k}\left(E\left(k_{r}\right)\right)\right)}
$$

if $A_{0}=D_{0}$ (cf. [2]). Moreover, we obtained the exact value of $\left(E(k): N_{k_{1} / k}\left(E\left(k_{1}\right)\right)\right)$ in [2]. Thus, we divide the situations into four cases. Let $d=d_{0}$ be the order of $\operatorname{cl}(\mathfrak{p})$.

1. The case $\left|D_{1}\right|=\left|D_{0}\right|$ (i.e., $\left.N_{k_{1} / k}\left(E\left(k_{1}\right)\right)=E(k)^{3}\right)$.

(A) If there exists an element $\alpha$ of $k_{2}$ such that $\mathfrak{p}_{2}^{d}=(\alpha)$, then $\left|D_{2}\right|=\left|D_{0}\right|$. Hence, $N_{k_{2} / k}\left(E\left(k_{2}\right)\right)=E(k)^{9}$ and $n_{2}^{(2)}=n_{2}+2$.

(B) If there exists a unit $\varepsilon$ of $k_{2}$ such that $N_{k_{2} / k}(\varepsilon)=\mu^{3}$, then $N_{k_{2} / k}\left(E\left(k_{2}\right)\right)$ $=E(k)^{3}$. Hence, $\left|D_{2}\right|=3\left|D_{0}\right|$ and $n_{2}^{(2)}=n_{2}+1$.

2. The case $\left|D_{1}\right|=3\left|D_{0}\right|$ (i.e., $\left.N_{k_{1} / k}\left(E\left(k_{1}\right)\right)=E(k)\right)$.

(C) If there exists an element $\alpha$ of $k_{2}$ such that $\mathfrak{p}_{2}^{3 d}=(\alpha)$, then $\left|D_{2}\right|=3\left|D_{0}\right|$. Hence, $N_{k_{2} / k}\left(E\left(k_{2}\right)\right)=E(k)^{3}$ and $n_{2}^{(2)}=n_{2}+1$. 
(D) If there exists a unit $\varepsilon$ of $k_{2}$ such that $N_{k_{2} / k}(\varepsilon)=\mu$, then $N_{k_{2} / k}\left(E\left(k_{2}\right)\right)$ $=E(k)$. Hence, $\left|D_{2}\right|=9\left|D_{0}\right|$ and $n_{2}^{(2)}=n_{2}$.

We search suitable elements of $k_{2}$ with the methods explained below, assuming that $E_{R}$ has a Galois generator $\varphi$. We shall explain in the next section how to find a candidate of $\varphi$. But we may disregard whether $E_{R}$ has a Galois generator if we have found the desired elements. Note that we obtain a generator of $\mathfrak{p}_{2}^{d_{2}}$ and are able to determine $n_{0}^{(2)}$ in each case.

Now assume that $E_{R}$ has a Galois generator $\varphi$. Then the following proposition handles the case $(\mathrm{D})$.

Proposition 4.1. We have $N_{k_{2} / k}\left(E\left(k_{2}\right)\right)=E(k)$ if and only if $\mu \Phi^{i} \in k_{2}^{9}$ for some $0 \leq i \leq 8$.

Proof. Assume that there exists $\varepsilon \in E\left(k_{2}\right)$ such that $N_{k_{2} / k}(\varepsilon)=\mu$. Then $\eta=$ $\varepsilon^{18} \tau^{-9} \mu^{-2} \in E_{R}$, where $\tau=N_{k_{2} / \mathbb{Q}_{2}}(\varepsilon)$. Since $\eta^{1+\sigma}= \pm\left(\varepsilon^{2} \tau^{-1}\right)^{9(1+\sigma)} \in E_{R}^{9}$, we have $\eta \equiv \Phi^{i}\left(\bmod E_{R}^{9}\right)$ for some $i$ from Lemma 2.3. Thus, we see that $\mu^{2} \Phi^{i} \in k_{2}^{9}$. Conversely, if $\mu \Phi^{i} \in k_{2}^{9}$, then there exists $\mu_{2} \in k_{2}$ such that $\mu_{2}^{9}=\mu \Phi^{i}$. Then $\mu_{2}$ is a unit of $k_{2}$ and $N_{k_{2} / k}\left(\mu_{2}\right)^{9}= \pm \mu^{9}$. Since $k$ is real and 9 is odd, we have $N_{k_{2} / k}\left(\mu_{2}\right)= \pm \mu$.

The case $(\mathrm{A})$ is handled by the next proposition.

Proposition 4.2. Assume that $A_{0}=D_{0}$. Let $d$ be the order of $\mathrm{cl}(\mathfrak{p})$ and take a generator $\alpha \in k$ of $\mathfrak{p}^{d}$. Then $\mathfrak{p}_{2}^{d}$ is principal if and only if $\alpha \Theta^{d} \mu^{i} \Phi^{j} \in k_{2}^{9}$ for some $0 \leq i, j \leq 8$ such that $j \not \equiv 0(\bmod 3)$.

Proof. Note that $\alpha^{1+\sigma}= \pm 3^{d}$. Assume that $\mathfrak{p}_{2}^{d}$ is principal and take a generator $\beta_{2} \in k_{2}$ of $\mathfrak{p}_{2}^{d}$. Then $\left(\beta_{2}^{9}\right)=\mathfrak{p}_{2}^{9 d}=\mathfrak{p}^{d}=(\alpha)$. Hence, $\beta_{2}^{9}=\alpha \varepsilon$ for some $\varepsilon \in E\left(k_{2}\right)$. Since $A_{0}=D_{0}$, the fact that $\mathfrak{p}_{2}^{d}$ is principal implies that $N_{k_{2} / k}\left(E\left(k_{2}\right)\right)=E(k)^{9}$. Put $N_{k_{2} / \mathbb{Q}_{2}}(\varepsilon)=\tau$ and $N_{k_{2} / k}(\varepsilon)= \pm \mu^{9 i}$ with suitable integer $i$. Then $\eta=\varepsilon^{2} \tau^{-1} \mu^{-2 i} \in$ $E_{R}$ and $\alpha^{2} \tau \mu^{2 i} \eta \in E_{R}^{9}$. Taking the norm from $k_{2}$ to $\mathbb{Q}_{2}$, we see that $3^{2 d} \tau^{2} \in \mathbb{Q}_{2}^{9}$ and hence $\tau \Theta^{-2 d} \in \mathbb{Q}_{2}^{9}$ from Corollary 3.2. Therefore, $\alpha^{2} \Theta^{2 d} \mu^{2 i} \eta \in k_{2}^{9}$. Since $\left(\alpha \Theta^{d}\right)^{1+\sigma}= \pm 3^{d} \Theta^{d(1+\sigma)} \equiv \Theta^{-d(1-\sigma)}\left(\bmod E\left(\mathbb{Q}_{2}\right)^{9}\right)$, we have $\left(\alpha \Theta^{d}\right)^{1+\sigma} \in E\left(\mathbb{Q}_{2}\right)^{9}$ from Lemma 3.3. Therefore, we see that $\eta^{1+\sigma} \in E_{R}^{9}$ and $\eta \equiv \Phi^{2 j}\left(\bmod E_{R}^{9}\right)$ with suitable $j$ from Lemma 2.3. Therefore, $\alpha^{2} \Theta^{2 d} \mu^{2 i} \Phi^{2 j} \in k_{2}^{9}$, and hence $\alpha \Theta^{d} \mu^{i} \Phi^{j} \in k_{2}^{9}$ because 2 is prime to 9 . Now assume that $j \equiv 0(\bmod 3)$; then $\alpha \Theta^{d} \mu^{i} \in k_{2}^{3}$. If we put $\beta=\alpha \mu^{i}$, then we see that $\beta^{1-\sigma} \in k_{2}^{3}$ from Lemma 3.3, and hence $\beta^{1-\sigma}=\gamma^{3}$ for some $\gamma \in k$ because $k$ is real. Then $\left(\mathfrak{p}^{1-\sigma}\right)^{d}=\left(\alpha^{1-\sigma}\right)=\left(\beta^{1-\sigma}\right)=(\gamma)^{3}$ implies that 3 divides $d$. Thus, from $\beta 3^{d}= \pm \beta \alpha^{1+\sigma}= \pm \beta \beta^{1+\sigma}= \pm\left(\beta \gamma^{-1}\right)^{3}$, we can write $\beta=\delta^{3}$ for some $\delta \in k$. Then we have $\mathfrak{p}^{d}=(\alpha)=(\beta)=(\delta)^{3}$, and hence $\mathfrak{p}^{d / 3}=(\delta)$, which contradicts the fact that $d$ is the order of $\operatorname{cl}(\mathfrak{p})$. Conversely, if $\alpha \Theta^{d} \mu^{i} \Phi^{j}=\alpha_{2}^{9}$ with $a_{2} \in k_{2}$, then $\mathfrak{p}_{2}^{9 d}=\mathfrak{p}^{d}=(\alpha)=\left(\alpha_{2}\right)^{9}$ and hence $\mathfrak{p}_{2}^{d}=\left(\alpha_{2}\right)$.

In the actual calculations, we expand $i$ and $j$ in 3-adic forms. Namely, we first get $\alpha_{1}=\left(\alpha \Theta^{d} \mu^{i_{1}} \Phi^{j_{1}}\right)^{1 / 3} \in k_{2}$ with $0 \leq i_{1} \leq 2,1 \leq j_{1} \leq 2$ and next get $\alpha_{2}=\left(\alpha_{1} \mu^{i_{2}} \Phi^{j_{2}}\right)^{1 / 3} \in k_{2}$ with $0 \leq i_{2}, j_{2} \leq 2$. In this manner, we can get a generator of $\mathfrak{p}_{2}^{d}$ within 15 trials if $\mathfrak{p}_{2}^{d}$ is principal.

The cases (B) and (C) are handled by the following propositions. We can prove these in the same manner as Propositions 4.1 and 4.2. So we omit the proofs. 
Proposition 4.3. We have $N_{k_{2} / k}\left(E\left(k_{2}\right)\right) \supset E(k)^{3}$ if and only if $\mu \Phi^{i} \in k_{2}^{3}$ for some $0 \leq i \leq 2$. Moreover, if we put $\mu_{1}^{3}=\mu \Phi^{i}$ with $\mu_{1} \in k_{2}$, then $N_{k_{2} / \mathbb{Q}_{2}}\left(\mu_{1}\right)= \pm 1$, $N_{k_{2} / k}\left(\mu_{1}\right)= \pm \mu^{3}$ and $\mu_{1}^{1+\sigma} \in k_{2}^{3}$.

Proposition 4.4. Assume that $N_{k_{1} / k}\left(E\left(k_{1}\right)\right)=E(k)$ and $A_{0}=D_{0}$. Let $d$ be the order of $\operatorname{cl}(\mathfrak{p})$ and take a generator $\alpha \in k$ of $\mathfrak{p}^{d}$. Let $\mu_{1} \in k_{2}$ be the element stated in Proposition 4.3. Then $\mathfrak{p}_{2}^{3 d}$ is principal if and only if $\alpha \Theta^{d} \mu_{1}^{i} \Phi^{j} \in k_{2}^{3}$ for some $0 \leq i, j \leq 2$.

\section{Galois generator of $E_{R}$}

In order to find a Galois generator $\varphi$ of $E_{R}$, we use Hasse's cyclotomic unit defined in [4, p.14]. We recall the definition. Let $K$ be a real abelian number field of conductor $f$ and $H$ the subgroup of $(\mathbb{Z} / f \mathbb{Z})^{\times}$corresponding to $K$. Then $-1+f \mathbb{Z} \in H$ because $K$ is real. Choose an odd representative from each pair $h,-h \in H$. Namely, let

$$
X= \begin{cases}\{1 \leq x \leq f \mid x: \text { odd }, x+f \mathbb{Z} \in H\} & \text { if } f \text { is odd } \\ \{1 \leq x \leq f / 2 \mid x: \text { odd }, x+f \mathbb{Z} \in H\} & \text { if } f \text { is even }\end{cases}
$$

Then, Hasse's unit is defined to be

$$
\xi=\prod_{x \in X}\left(\zeta_{2 f}^{x}-\zeta_{2 f}^{-x}\right)
$$

where $\zeta_{2 f}$ denotes a primitive $(2 f)$ th root of unity. In general, $\xi$ is neither a unit nor contained in $K$. But in our case, namely in the case $K=k_{2}$, we verified that $\xi \in E\left(k_{2}\right)$ and moreover that $N_{k_{2} / k}(\xi)= \pm 1$ by a numerical calculation. Therefore, if we put $\eta=\xi^{2} N_{k_{2} / \mathbb{Q}_{2}}(\xi)^{-1}$, then $\eta \in E_{R}$. Now assume that $E_{R}$ has a Galois generator $\varphi$. Then $\eta$ can be represented as $\eta_{0}= \pm \varphi_{0}^{e_{0}} \varphi_{1}^{e_{1}} \cdots \varphi_{7}^{e_{7}}$ with suitable integers $e_{i}$. Applying $\sigma$ seven times on this relation, we have eight relations between $\eta_{i}$ and $\varphi_{i}$, which we consider the equation of $\varphi_{i}$. We solve this equation for each pair $\left(e_{0}, e_{1}, \ldots, e_{7}\right)$. If we see $\varphi \in k_{2}$ for some $\left(e_{0}, e_{1}, \ldots, e_{7}\right)$, then we consider this $\varphi$ as a candidate of a Galois generator and pursue the calculation with the algorithms in $\S 4$.

\section{Capitulation problem}

We studied Greenberg's conjecture mainly in the case $A_{0}=D_{0}$ in [2]. When $A_{0} \neq D_{0}$, we consider the conjecture by relating it to a capitulation problem. Let $i_{0, r}$ be the inclusion map from $k$ to $k_{r}$.

Lemma 6.1. Let $k$ be a real quadratic field and $p$ an odd prime number which splits in $k$. Assume that $n_{2}=1$ and $i_{0, r}\left(A_{0}\right) \subset D_{r}$ for some $r \geq 0$. Then $\lambda_{p}(k)=$ $\mu_{p}(k)=0$.

Proof. Let $k_{\infty} / k$ be the cyclotomic $\mathbb{Z}_{p}$-extension of $k$. Let $B_{r}$ be the subgroup of $A_{r}$ invariant under $G\left(k_{\infty} / k\right)$, and $B_{r}^{\prime}$ the subgroup of $B_{r}$ consisting of elements which contain an ideal invariant under $G\left(k_{\infty} / k\right)$. Then $B_{r}^{\prime}=i_{0, r}\left(A_{0}\right) D_{r}$ and

$$
\left|B_{r}^{\prime}\right|=\left|A_{0}\right| \frac{p^{r}}{\left(E(k): N_{k_{r} / k}\left(E\left(k_{r}\right)\right)\right)}
$$

from genus theory. The assumption $i_{0, r}\left(A_{0}\right) \subset D_{r}$ implies $B_{r}^{\prime}=D_{r}$, and hence the assumption $n_{2}=1$ and (1) yields $\left|D_{r}\right|=\left|A_{0}\right|$. On the other hand, we have 
$\left|B_{n}\right|=\left|A_{0}\right|$ for all $n \geq 0$ from Lemma 2.2 in [2]. Therefore, $B_{n}=D_{n}$ for all $n \geq r$, and hence $\lambda_{p}(k)=0$ from Theorem 2 in [3].

There are six $k$ 's in Table 1 of [2] such that $A_{0} \neq D_{0}$ and $\lambda_{3}(k)$ is not known, namely $k=\mathbb{Q}(\sqrt{m})$ where $m=2713,3739,5938,7726,8017$ and 8782. For these $k$ 's, we know that $\left|A_{0}\right|=3,\left|D_{0}\right|=\left|D_{1}\right|=1$ and $\left(E(k): N_{K_{1} / k}\left(E\left(k_{1}\right)\right)\right)=3$. Hence, $\left|i_{0,1}\left(A_{0}\right)\right|=3$. So we need consider $i_{0,2}\left(A_{0}\right)$. For $\mathbb{Q}(\sqrt{3739})$ and $\mathbb{Q}(\sqrt{5938})$, we could find a generator of $\mathfrak{p}_{2}^{d}$, where $d$ is the order of $\operatorname{cl}(\mathfrak{p})$. Therefore, we have $\left|D_{2}\right|=1$ and $\left|i_{0,2}\left(A_{0}\right)\right|=3$. For $\mathbb{Q}(\sqrt{7726})$, we could not find a Galois generator $\varphi$ of $E_{R}$. For the remaining three $k$ 's, we found candidates of $\varphi$, but could not find a generator of $\mathfrak{p}_{2}^{d}$. Thus, $\left|D_{2}\right|$ seems to be 3 and there is a possibility of $i_{0,2}\left(A_{0}\right) \subset D_{2}$. The following lemma allows us to verify this possibility. It assumes again the existence of $\varphi$. But we may disregard it if we found the desired element as explained in $\S 4$.

Lemma 6.2. Assume that $\left|A_{0}\right|=3,\left|D_{0}\right|=\left|D_{1}\right|=1$ and $\left(E(k): N_{k_{1} / k}\left(E\left(k_{1}\right)\right)\right)=$ 3. Let $\mathfrak{q}$ be a nonprincipal ideal of $k$ such that $\mathfrak{q}^{3}=(\beta)$ for some $\beta \in k$. Let $\mathfrak{p}^{d}=(\alpha)$ with $\alpha \in k$, where $d$ is the order of $\operatorname{cl}(\mathfrak{p})$. Then $i_{0,2}\left(A_{0}\right) \subset D_{2}$ if and only if $\beta^{3} \alpha^{e} \Theta^{e d} \mu^{i} \Phi^{j} \in k_{2}^{9}$ for some $0 \leq e \leq 2$ and $0 \leq i, j \leq 8$. Moreover, $i_{0,2}\left(A_{0}\right)=1$ if and only if $e=0$.

Proof. Assume that $i_{0,2}\left(A_{0}\right) \subset D_{2}$. Then $B_{2}^{\prime}=D_{2}$. Since

$$
\left|B_{2}^{\prime}\right|=\left|A_{0}\right| \frac{p^{2}}{\left(E(k): N_{k_{2} / k}\left(E\left(k_{2}\right)\right)\right)} \geq\left|A_{0}\right|=3,
$$

we have $\left|B_{2}^{\prime}\right|=\left|D_{2}\right|=3$, and hence $\left(E(k): N_{k_{2} / k}\left(E\left(k_{2}\right)\right)\right)=9$. Since $i_{0,2}\left(A_{0}\right) \subset$ $D_{2}$, we see that $\mathfrak{q p}_{2}^{e}$ is principal in $k_{2}$ for some $0 \leq e \leq 2$, and hence $\mathfrak{q}^{9} \mathfrak{p}_{2}^{9 e}=$ $\left(\beta^{3} \alpha^{e}\right)=\left(\gamma^{9}\right)$ for some $\gamma \in k_{2}$. Therefore, $\beta^{3} \alpha^{e} \varepsilon \in k_{2}^{9}$ for some $\varepsilon \in E\left(k_{2}\right)$. We can see that $\varepsilon \equiv \Theta^{e d} \mu^{i} \Phi^{j}\left(\bmod E\left(k_{2}\right)^{9}\right)$ for some $0 \leq i, j \leq 8$ in the same way as in the proof of Proposition 4.2. Conversely, assume that $\beta^{3} \alpha^{e} \Theta^{e d} \mu^{i} \Phi^{j}=\gamma^{9}$ with $\gamma \in k_{2}$. Then $\mathfrak{q}^{9} \mathfrak{p}_{2}^{9 e}=(\gamma)^{9}$, and hence $\mathfrak{q}=\mathfrak{p}_{2}^{-e}(\gamma)$. Hence, we have proved the first assertion. The second is easy.

For $k=\mathbb{Q}(\sqrt{2713}), \mathbb{Q}(\sqrt{8017})$ and $\mathbb{Q}(\sqrt{8782})$, we verified that $i_{0,2}\left(A_{0}\right)=D_{2}$ by Lemma 6.2. So we see that $\lambda_{3}(k)=0$ by Lemma 6.1 and moreover that $\left|D_{2}\right|=3$ and $\left(E(k): N_{k_{2} / k}\left(E\left(k_{2}\right)\right)\right)=9$ by a trivial argument.

\section{Computational technique}

In this section, we explain a technique of calculation using a computer. Let $\theta=\cos (2 \pi / 27)$ and

$$
\omega= \begin{cases}\sqrt{m} & \text { if } m \equiv 2,3 \quad(\bmod 4), \\ (1+\sqrt{m}) / 2 & \text { if } m \equiv 1 \quad(\bmod 4)\end{cases}
$$

for a positive square-free integer $m$. Then

$$
\left\{1, \theta, \theta^{2}, \ldots, \theta^{8}, \omega, \omega \theta, \omega \theta^{2}, \ldots, \omega \theta^{8}\right\}
$$

forms a $\mathbb{Z}$-basis of the integer ring of $k_{2}=\mathbb{Q}(\theta, \sqrt{m})$. The coefficients $x_{i} \in \mathbb{Z}$ of Hasse's unit $\xi$ with respect to this basis are obtained by solving approximately the linear equations made up from the conjugates of

$$
x_{0}+x_{1} \theta+\cdots+x_{8} \theta^{8}+x_{9} \omega+x_{10} \omega \theta \cdots+x_{17} \omega \theta^{8}=\xi .
$$


Here the conjugates are taken with respect to the generator $\sigma$ of $G\left(k_{2} / \mathbb{Q}\right)$ such that $\theta^{\sigma}=\cos (4 \pi / 27)$ and $\sqrt{m}^{\sigma}=-\sqrt{m}$, and the approximate value of $\xi_{i}$ is calculated from

$$
\xi_{i}=(-1)^{\ell} \prod_{x \in X}\left(2 \sin \left(\frac{s^{i} x \pi}{f}\right)\right)
$$

where $2 \ell=|X|, f$ is the conductor of $k_{2}$ and $s$ is an integer such that $s \equiv 2$ $(\bmod 27)$ and $\chi(s)=-1$ for the character $\chi$ of $\mathbb{Q}(\sqrt{m})$. We first calculate the logarithm of the absolute value of (3) with a 64-bit floating-point number and know the necessary precision for this product. Then we proceed with a suitable precision.

Next we have to represent a conjugate of an integer of $k_{2}$ and a product of integers of $k_{2}$ in the basis (2). To do so, we have to represent a conjugate of an integer of $\mathbb{Q}_{2}$ and a product of integers of $\mathbb{Q}_{2}$ with respect to $\left\{1, \theta, \theta^{2}, \ldots, \theta^{8}\right\}$. This is easily done by computing

$$
A^{-1}\left(\begin{array}{cccc}
\theta_{1} & \theta_{1}^{2} & \cdots & \theta_{1}^{8} \\
\theta_{2} & \theta_{2}^{2} & \cdots & \theta_{2}^{8} \\
\vdots & \vdots & \ddots & \vdots \\
\theta_{9} & \theta_{9}^{2} & \cdots & \theta_{9}^{8}
\end{array}\right) \quad \text { and } \quad A^{-1}\left(\begin{array}{cccc}
\theta_{0}^{9} & \theta_{0}^{10} & \cdots & \theta_{0}^{16} \\
\theta_{1}^{9} & \theta_{1}^{10} & \cdots & \theta_{1}^{16} \\
\vdots & \vdots & \ddots & \vdots \\
\theta_{8}^{9} & \theta_{8}^{10} & \cdots & \theta_{8}^{16}
\end{array}\right)
$$

where $A=\left(\theta_{i}^{j}\right)_{0 \leq i, j \leq 8}$.

Finally, we have to check whether an integer $\alpha$ of $k_{2}$ represented in (2) is a cube in $k_{2}$. This is routine work. Namely, we first calculate the approximate value of $\alpha_{0}^{1 / 3}+\alpha_{1}^{1 / 3}+\cdots+\alpha_{17}^{1 / 3}$. If this is not an integer, then $\alpha$ is not a cube. If it is close to a natural integer, we obtain coefficients by solving the linear equations involving $\alpha_{i}^{1 / 3}$. If all the coefficients are close to natural integers, then we round them to integers and get $\beta \in k_{2}$ with these integral coefficients. We compare $\beta^{3}$ with $\alpha$. If $\beta^{3}=\alpha$, then $\alpha$ is a cube in $k_{2}$.

\section{EXAMPLES}

We executed the calculations for $52 \mathrm{k}$ 's stated in $\S 1$ with the method in the preceding sections. We found a candidate of a Galois generator $\varphi$ for $48 k$ 's. Namely, we could find the desired element for $(\mathrm{A})-(\mathrm{D})$ and could determine $n_{0}^{(2)}$ and $n_{2}^{(2)}$ for $48 k$ 's. There are $29 k$ 's which satisfy $A_{0}=D_{0}$ and $n_{0}^{(2)}=3$. For these $k$ 's, we see that $\lambda_{3}(k)=0$ from Theorem 2 in $[2]$. For $k=\mathbb{Q}(\sqrt{2149})$ and $\mathbb{Q}(\sqrt{4081})$, we have $3<n_{0}^{(2)}<n_{2}^{(2)}$, and hence conclude that $\lambda_{3}(k)=0$ from Theorem 1 in [2]. Therefore, together with the three $k$ 's in $\S 6$, we obtained $34 k$ 's which satisfy $\lambda_{3}(k)=0$.

We can determine $\left|A_{2}\right|$ in some cases using Lemma 2.3 in [1]. Moreover, we can apply a similar argument for $m=3739$.

We shall summarize our computational results in Table 1 . Here, $\lambda_{3}^{+}$denotes $\lambda_{3}(k)$ and $\lambda_{3}^{-}$denotes the minus part of the $\lambda_{3}$-invariants of $k^{*}=k\left(\zeta_{3}\right)$. The asterisks mean that we do not know the value. A 64-bit work station DEC3000/300AXP with $\mathrm{C}$ language did the computations in one day. 


\section{TABLE 1}

\begin{tabular}{|c|c|c|c|c|c|c|c|c|c|c|c|c|c|c|c|}
\hline$m$ & $n_{0}$ & $n_{2}$ & $n_{0}^{(1)}$ & $n_{2}^{(1)}$ & $n_{0}^{(2)}$ & $n_{2}^{(2)}$ & $\left|D_{0}\right|$ & $\left|A_{0}\right|$ & $\left|D_{1}\right|$ & $\left|A_{1}\right|$ & $\left|D_{2}\right|$ & $\left|A_{2}\right|$ & $i_{0,2}\left(A_{0}\right)$ & $\lambda_{3}^{-}$ & $\lambda_{3}^{+}$ \\
\hline 295 & 2 & 2 & 3 & 3 & 3 & 4 & 1 & 1 & 1 & 3 & 1 & 9 & & 1 & 0 \\
\hline 397 & 2 & 2 & 3 & 3 & 3 & 4 & 1 & 1 & 1 & 3 & 1 & 9 & & 1 & 0 \\
\hline 727 & 2 & 3 & 3 & 3 & 3 & 4 & 1 & 1 & 3 & 9 & 3 & $*$ & & 2 & 0 \\
\hline 745 & 2 & 2 & 3 & 3 & 3 & 4 & 1 & 1 & 1 & 3 & 1 & 9 & & 1 & 0 \\
\hline 1714 & 2 & 2 & 3 & 3 & 3 & 4 & 3 & 3 & 3 & 9 & 3 & * & & 4 & 0 \\
\hline 1738 & 2 & 2 & 3 & 3 & 4 & 4 & 1 & 1 & 1 & 3 & 1 & 9 & & 1 & * \\
\hline 2029 & 2 & 2 & 3 & 3 & 3 & 4 & 1 & 1 & 1 & 3 & 1 & 9 & & 1 & 0 \\
\hline 2059 & 3 & 3 & 4 & 4 & 5 & 5 & 1 & 1 & 1 & 3 & 1 & 9 & & 1 & * \\
\hline 2149 & 4 & 4 & 5 & 5 & 5 & 6 & 1 & 1 & 1 & 3 & 1 & 9 & & 1 & 0 \\
\hline 2713 & 1 & 1 & 2 & 2 & 3 & 3 & 1 & 3 & 1 & 9 & 3 & * & $=D_{2}$ & 1 & 0 \\
\hline 2794 & 2 & 3 & 3 & 3 & 3 & 3 & 1 & 1 & 3 & 9 & 9 & $*$ & & 2 & 0 \\
\hline 2917 & 3 & 3 & 4 & 4 & 4 & 5 & 3 & 3 & 3 & 9 & 3 & * & & 3 & * \\
\hline 3469 & 2 & 2 & 3 & 3 & * & * & 1 & 1 & 1 & 9 & * & * & & 2 & $*$ \\
\hline 3490 & 2 & 2 & 3 & 3 & 4 & 4 & 1 & 1 & 1 & 3 & 1 & 9 & & 1 & $*$ \\
\hline 3739 & 2 & 2 & 3 & 3 & 4 & 4 & 1 & 3 & 1 & 9 & 1 & 27 & $\neq D_{2}$ & 1 & * \\
\hline 4081 & 3 & 3 & 4 & 4 & 4 & 5 & 1 & 1 & 1 & 3 & 1 & 9 & & 1 & 0 \\
\hline 4279 & 3 & 3 & 3 & 3 & 3 & 3 & 3 & 3 & 9 & 27 & 27 & $*$ & & 2 & 0 \\
\hline 4654 & 2 & 2 & 3 & 3 & 3 & 4 & 1 & 1 & 1 & 3 & 1 & 9 & & 1 & 0 \\
\hline 4741 & 2 & 3 & 3 & 3 & 3 & 3 & 1 & 1 & 3 & 9 & 9 & $*$ & & 3 & 0 \\
\hline 4789 & 2 & 2 & 3 & 3 & 4 & 4 & 1 & 1 & 1 & 3 & 1 & 9 & & 1 & * \\
\hline 5185 & 2 & 2 & 3 & 3 & 3 & 4 & 1 & 1 & 1 & 3 & 1 & 9 & & 1 & 0 \\
\hline 5530 & 2 & 2 & 3 & 3 & 3 & 4 & 1 & 1 & 1 & 9 & 1 & $*$ & & 2 & 0 \\
\hline 5533 & 2 & 3 & 3 & 3 & 4 & 4 & 1 & 1 & 3 & 9 & 3 & * & & 2 & * \\
\hline 5611 & 3 & 3 & 3 & 3 & 3 & 4 & 1 & 1 & 3 & 9 & 3 & $*$ & & 3 & 0 \\
\hline 5938 & 1 & 1 & 2 & 2 & 3 & 3 & 1 & 3 & 1 & 9 & 1 & $*$ & $\neq D_{2}$ & 1 & * \\
\hline 5971 & 2 & 3 & 3 & 3 & * & $*$ & 1 & 1 & 3 & 27 & * & $*$ & & 3 & $*$ \\
\hline 6169 & 2 & 2 & 3 & 3 & 3 & 4 & 1 & 1 & 1 & 3 & 1 & 9 & & 1 & 0 \\
\hline 6187 & 2 & 2 & 3 & 3 & * & $*$ & 1 & 1 & 1 & 9 & * & * & & 3 & $*$ \\
\hline 6202 & 2 & 2 & 3 & 3 & 3 & 4 & 1 & 1 & 1 & 3 & 1 & 9 & & 1 & 0 \\
\hline 6271 & 2 & 2 & 3 & 3 & 3 & 4 & 1 & 1 & 1 & 3 & 1 & 9 & & 1 & 0 \\
\hline 6286 & 2 & 2 & 3 & 3 & 3 & 4 & 1 & 1 & 1 & 3 & 1 & 9 & & 1 & 0 \\
\hline 6559 & 2 & 4 & 3 & 4 & 3 & 5 & 9 & 9 & 27 & 81 & 27 & $*$ & & 2 & 0 \\
\hline 6871 & 2 & 2 & 3 & 3 & 3 & 4 & 1 & 1 & 1 & 3 & 1 & 9 & & 1 & 0 \\
\hline 6934 & 2 & 2 & 3 & 3 & 3 & 4 & 1 & 1 & 1 & 3 & 1 & 9 & & 1 & 0 \\
\hline 7006 & 3 & 3 & 3 & 4 & 3 & 4 & 3 & 3 & 3 & 9 & 3 & $*$ & & 3 & 0 \\
\hline 7309 & 2 & 2 & 3 & 3 & 4 & 4 & 1 & 1 & 1 & 3 & 1 & 9 & & 1 & $*$ \\
\hline 7321 & 2 & 2 & 3 & 3 & 4 & 4 & 1 & 1 & 1 & 3 & 1 & 9 & & 1 & $*$ \\
\hline 7429 & 2 & 3 & 3 & 3 & 3 & 3 & 1 & 1 & 3 & 9 & 9 & $*$ & & 2 & 0 \\
\hline 7465 & 3 & 3 & 3 & 4 & 3 & 5 & 9 & 9 & 9 & 27 & 9 & * & & 2 & 0 \\
\hline 7582 & 2 & 2 & 3 & 3 & 4 & 4 & 1 & 1 & 1 & 3 & 1 & 9 & & 1 & $*$ \\
\hline 7642 & 2 & 3 & 3 & 3 & 4 & 4 & 1 & 1 & 3 & 9 & 3 & $*$ & & 2 & $*$ \\
\hline 7726 & 2 & 2 & 2 & 3 & $*$ & $*$ & 1 & 3 & 1 & 81 & * & * & & 3 & * \\
\hline 7957 & 2 & 2 & 3 & 3 & 3 & 4 & 1 & 1 & 1 & 3 & 1 & 9 & & 1 & 0 \\
\hline 8017 & 1 & 1 & 2 & 2 & 3 & 3 & 1 & 3 & 1 & 9 & 3 & $*$ & $=D_{2}$ & 1 & 0 \\
\hline 8101 & 2 & 2 & 3 & 3 & 4 & 4 & 1 & 1 & 1 & 3 & 1 & 9 & & 1 & $*$ \\
\hline 8155 & 2 & 2 & 3 & 3 & 3 & 4 & 1 & 1 & 1 & 3 & 1 & 9 & & 1 & 0 \\
\hline 8569 & 2 & 2 & 3 & 3 & 3 & 4 & 1 & 1 & 1 & 3 & 1 & 9 & & 1 & 0 \\
\hline 8782 & 1 & 1 & 2 & 2 & 3 & 3 & 1 & 3 & 1 & 9 & 3 & $*$ & $=D_{2}$ & 1 & 0 \\
\hline 9058 & 2 & 2 & 3 & 3 & 3 & 4 & 1 & 1 & 1 & 3 & 1 & 9 & & 1 & 0 \\
\hline 9634 & 3 & 4 & 3 & 5 & 3 & 6 & 3 & 3 & 3 & $y$ & 3 & $*$ & & 2 & 0 \\
\hline 9691 & 2 & 3 & 3 & 3 & 3 & 3 & 1 & 1 & 3 & 9 & 9 & $*$ & & 2 & 0 \\
\hline 9814 & 4 & 4 & 5 & 5 & 6 & 6 & 1 & 1 & 1 & 3 & 1 & 9 & & 1 & * \\
\hline
\end{tabular}

\section{REFERENCES}

1. T. Fukuda, Iwasawa's $\lambda$-invariants of certain real quadratic fields, Proc. Japan Acad. 65, (1989), 260-262. MR 91b:11115

2. T. Fukuda and H. Taya, The Iwasawa $\lambda$-invariants of $\mathbb{Z}_{p}$-extensions of real quadratic fields, Acta Arith. 69 (1995), 277-292.

3. R. Greenberg, On the Iwasawa invariants of totally real number fields, Amer. J. Math. 98 (1976), 263-284. MR 53:5529

4. H. Hasse, Über die Klassenzahl abelscher Zahlkörper, Akademie Verlag, Berlin, 1952. MR 14:141a 
5. S. Mäki, The determination of units in real cyclic sextic fields, Lecture Notes in Math., vol. 797, Springer-Verlag, Berlin, Heidelberg, New York, 1980. MR 82a:12004

6. H. Taya, Computation of $\mathbb{Z}_{3}$-invariants of real quadratic fields, Math. Comp. 65 (1996), 779 784. CMP 95:13

Department of Mathematics, College of Industrial Technology, Nihon University, 2-11-1 Shin-ei, Narashino, Chiba, Japan

E-mail address: fukuda@math.cit.nihon-u.ac.jp 